\title{
Water on Planets
}

\author{
James F Bell III \\ Dept. of Astronomy, Cornell University, 402 Space Sciences Bldg., Ithaca NY 14853 USA \\ email: jfb8@cornell.edu
}

\begin{abstract}
Water is an abundant molecule in the Cosmos. It has exploitable and unique spectroscopic and physical properties and has been found to be ubiquitous in places that we would expect in the standard model of solar system formation and nebular condensation: beyond the snow line in outer solar system planets, moons, asteroids, and comets. However, water is also an important constituent of planetary bodies (dominating at least one of their surfaces) in the inner solar system, likely indicating significant mixing between inner and outer solar system reservoirs of water during planetary accretion and the early history of the solar system. Water has played a critical role in the differential evolution of the terrestrial planets Venus, Earth, and Mars, and the concept of the "habitable zone" where liquid water could be stable on an Earth-like planet provides a starting point for assessing the habitability of worlds in our solar system and beyond. Examples of potentially habitable environments outside this zone in our own solar system warn us that this concept should only be a guide, however-important exceptions will no doubt occur. Recent discoveries of past liquid water and abundant present subsurface ice on Mars, of water reservoirs in unexpected places like the poles of Mercury and the Moon and the subsurface of Enceladus, of water in circumstellar disks and in the atmospheres of extrasolar planets, and the expectation of the discovery of water on Earth-like worlds in the habitable zones around other stars make this an exciting time in the study of water on planets both in our own solar system, and beyond.
\end{abstract}

Keywords. water, planets, satellites, asteroids, comets, extrasolar planets, habitable zone

\section{Introduction}

The topic of "water on planets" covers an expansive, literally astronomical, range of objects and scientific realms. Aspects of physics, chemistry, astronomy, geology, and even biology all come into play when considering the origin and influence of water on the history and evolution of planetary (and satellite, asteroidal, cometary, and even dust grain) surfaces, interiors, and atmospheres. Outstanding reviews of this topic have been presented recently by Chambers, 2004, Drake, 2005, Encrenaz, 2008, Owen, 2008, and Albarede, 2009.

Any review of this broad topic is almost certain to be biased by the reviewer's own background, experience, and perception of the role of water in their own specific scientific studies. My own bias is to approach this topic with the perspective of a geologist. That is, on one level, to think about water on planets in a forensic sense: what clues can we observe on planets or other solar system (or extrasolar) objects that can reveal the role, if any, that water plays or has played in the history of those bodies? On another level, a geologic approach to the study of water also includes a built-in need to understand not only the specific and quantitative local observations, but also the bigger-picture, qualitative context of water's influence on classes of planetary objects and/or regions of planetary and interplanetary space. Such a perspective lends itself to the classic "What, Where, When, and Why" approach to studying this topic. Perhaps this approach is a bit cliché, but for as broad a topic as water on planets it can provide a useful framework within which to begin more specific, focused studies. 
Another bias that I tip-toed around above has to do with the definition of "planet." While the IAU has recently adopted a controversial new, dynamically-based definition (IAU, 2006), my own personal working "definition" focuses on the physical and/or geophysical properties of each object itself, rather than on its particular location or orbit. For example, if an object is large enough for its own gravity to have given it a rounded shape, for internal processes such as differentiation to have occurred, for internal gravitational and/or radioactive heat sources to have enabled some form of volcanism and possibly even volatile release to form an atmosphere-these kinds of characteristics define, for me, an object that I would call a "planet." Such a definition is perhaps not so uncommon among astronomers and planetary scientists (e.g., PSI, 2006; Sykes, 2009). More importantly, it enables a more thorough, complete review and understanding of the role and influence of water on a much wider sampling of solar system bodies than a narrow definition of "planets" would otherwise allow. Why, for example, should we consider Mercury a planet and not the comparable or larger (and more water-rich) bodies Callisto, Titan, and Ganymede? By this definition, our solar system has some 35 to 40 "planets" and likely even more yet to be discovered in the Kuiper Belt.

Having made these various confessions of biases, let us proceed to review and survey the exciting and topical study of water on planets. This review begins with an overview focused on the question of What is water? Specifically, what are the properties of the water molecule that make it a rich object of study as well as special from the standpoint of planetary and chemical/biologic processes. I then discuss the question of Where do we find water (in all its forms)? Thinking about this question in our own solar system as well as extrasolar planetary and protoplanetary systems will naturally lead to the introduction of key concepts like the snow line and the habitable zone. Next I digress a bit to the specific questions of When (and where) has water been liquid, and for how long. The digression is worth considering because the presence and stability of liquid water is a key requirement for assessing the potential habitability of worlds-especially Earth-like worlds. Finally, I conclude by wandering a bit into more philosophical territory and considering the Why. That is, I attempt to explain why, in both the scientific and sociological senses, we as astronomers, planetary scientists, educators, and general students of the Cosmos, must care about and aggressively pursue the study of water on planets.

\section{Consider the $\mathrm{H}_{2} \mathrm{O}$ molecule...}

Hydrogen and Oxygen are the \#1 and \#3 most abundant elements in the Universe, and so it should not be surprising that $\mathrm{H}_{2} \mathrm{O}$ is an abundant and ubiquitous molecule. $\mathrm{H}_{2} \mathrm{O}$ has a net dipole moment, meaning that it is endowed with interesting physical and chemical properties as well as some important exploitable properties from the perspective of remote sensing. For example, water is extremely spectroscopically active, exhibiting a strong and diagnostic fundamental mid-IR bending mode absorption as well as a family of overtone and combination bands in the near-IR. In the solid phase, these bands exhibit detectable variations in band strength, center, and shape that can be used to provide diagnostic information on the abundance, crystallinity, and temperature of water ice on planetary bodies, as well as the presence of impurities or exotic structural forms like clathrates (e.g., Clark, 1981; Warren, 1982; Grundy et al., 1999; de Bergh et al., 2008). In addition, the density of water's solid phase $\left(0.92 \mathrm{~g} / \mathrm{cm}^{3}\right)$ is less than the density of the liquid phase $\left(1.00 \mathrm{~g} / \mathrm{cm}^{3}\right)$. This is an unusual property among most molecules that might seem otherwise esoteric if not for the important fact that water ice floating on liquid water acts as a thermal insulator (a so-called "solid-state greenhouse"; e.g., Matson and Brown, 1989) and thus allows liquid water to remain stable under sub-freezing temperatures. 
Other aspects of water's physical- and phase-related properties are also important from the standpoint of considering its history and influence on planetary bodies. For example, the phase diagram of water reveals that the boundaries between solid, liquid, and gas phases span the range of typical terrestrial surface conditions, as well as current and potential past Martian surface conditions (see $\S 4$ ). Thus, phase changes and their resulting volumetric and/or enthalpic implications are expected to play important roles in the energy balance and the nature of geologic processes on planetary surfaces and interiors where water is a significant component. Increasing the salinity of water also has an important effect, lowering the freezing point significantly for saline solutions (like Earth's oceans) and thus potentially significantly expanding the temperature range over which water can remain liquid. We will come back to some of the key the implications of that particular behavior in $\S 3$ and $\S 4$. Finally, another property of water that is critical in the study of its history and influence on planetary bodies is that it is an excellent and ubiquitous solvent except for the so-called "CHNOPS"-based (Carbon, Hydrogen, Nitrogen, Oxygen, Phosphorus, and Sulfur) organic molecules. The fact that most of the organic molecules that are key to complex biochemical reactions and life as we know it do not dissolve in water provides a convenient medium for the transport of energy to and removal of byproducts from these molecules as part of the chemistry of life. We will return to this topic more in $\S 5$.

\section{Where do we find water?}

\subsection{The Snow Line}

Before embarking on a survey of where we find water in our solar system and beyond, it is instructive to ask, based on our current understanding of planetary formation and solar system evolution, where should we find water in a solar system? A key concept here is that of the "snow line." In the currently-accepted model of solar system formation that begins with a hot, rotating cloud of gas and dust that collapses to a warm accreting debris disk, the snow line is the distance from the central star beyond which a particular volatile molecular component condenses as ice as the disk cools (e.g., Cassen, 1994; Lecar et al., 2006). Based on modeling and observations of the heliocentric distance for the onset of cometary activity, the snow line for $\mathrm{H}_{2} \mathrm{O}$ in the solar system today is around 2.0-2.5 AU (e.g., at $160 \mathrm{~K} \leqslant \mathrm{~T} \leqslant 200 \mathrm{~K}$ approximately; see review in Encrenaz, 2008). The water snow line was likely to have been farther out (perhaps near 4-5 AU, near where proto-Jupiter formed) in the early solar system (e.g., Stevenson and Lunine, 1988; Encrenaz, 2008), and of course there are different, similarly more distant "snow" lines for molecular disk volatiles like $\mathrm{CH}_{4}, \mathrm{NH}_{3}, \mathrm{CO}_{2}$, and others that have lower condensation temperatures based on their saturation vapor pressure behavior.

The fact that water condenses at such a significantly higher temperature than other common nebula volatiles means that water is the first volatile phase to condense at a particular heliocentric distance. Combined with the ubiquity of water in general, this implies that water ice is likely to be the dominant volatile in a typical solar nebula, and that the snow line marks an important boundary in a typical solar system, beyond which significantly greater abundances of solids (ice) are available for planetary accretion and growth (e.g., Stevenson and Lunine, 1988; Encrenaz, 2008). The concept of a hard "line" beyond which water condenses is likely to be unrealistic, however, as local, synopticscale variations in nebular conditions (e.g., pressure, temperature, density, composition, grain size) almost certainly would have resulted in the water ice condensation occurring within a more fuzzy zone rather than at a specific, narrow boundary. Indeed, Podolak, 2009 has called for the use of the term "ice stability region" rather than "snow line" to 
acknowledge the complexities of the physics and chemistry occurring within condensing stellar and protostellar disks.

\subsection{Where do we expect to find water in our solar system?}

\subsubsection{Giant Planets.}

If the snow line (or ice stability region) concept is correct, then we would certainly expect to detect large abundances of water in the interiors and satellites of the giant planets of the outer solar system. Indeed, tropospheric water vapor has been detected from telescopic (e.g., KAO, ISO) and spacecraft (e.g., Voyager, Galileo) infrared spectroscopic observations of Jupiter and Saturn (see Encrenaz, 2008 for a review), which represent more than $91 \%$ of the planetary mass in our solar system. Interestingly, however, the measured relative abundances are lower than would be predicted from the saturation vapor pressure data alone, suggesting that other processes, perhaps such as cloud condensation, are influencing the ability of spectroscopic observations to accurately estimate the water inventories of the giant planets. Galileo probe measurements of water vapor in the Jovian atmosphere (e.g., Wong et al., 2004) confirmed in general the dryer-than-expected nature of the upper troposphere, but the probe's entry into what appears to have been an anomalous "hot spot" complicates the extrapolation of the probe's measurements to the troposphere (and interior) overall.

The next largest reservoir of planetary water should be within Uranus and Neptune $(7 \%$ of the planetary mass in our solar system). However, there are no data available on the abundance of tropospheric (0.1 -1.0 bar) or deeper water on these planets, despite models that predict tropospheric condensation (e.g., Atreya, 1986). ISO $~ 40 \mathrm{~m}$ infrared emission spectra do provide evidence for stratospheric $\left(10^{-3}\right.$ to 0.1 bar) water in all four giant planets, a rather surprising observation that was used to infer the existence of significant external sources of water to these planet's atmospheres (e.g., Feuchtgruber et al., 1997; Lellouch et al., 2002). Such a postulate should probably not be surprising, given what we now know may be relatively common impacts of volatile-rich comets with the giant planets, witnessed first so dramatically with the impact of split comet Shoemaker-Levy 9 with Jupiter in the summer of 1994, and most recently with the apparent impact (not directly observed) of a previously unknown comet with Jupiter in the summer of 2009. Given the large uncertainties in impactor flux and composition, it is probably not possible to accurately determine whether these kinds of small body impacts could explain the observed stratospheric abundances of water in the giant planets. Nonetheless, they are clearly a potentially important source of external volatiles, for the giant planets and, as we shall discuss below, for the terrestrial planets as well.

Given the dearth of actual measurements, modeling studies continue to provide the best way to estimate the water inventory, composition, and other aspects of the interiors of the giant planets. Canonical giant planet interior models predict relatively small (e.g., $10 \%$ RJ) refractory cores surrounded by ices mixed throughout the deep atmosphere (e.g., Pollack et al., 1996). More recent models, employing new measurement constraints from telescopic and Galileo mission probe and gravity data, hypothesize that the ices are not particularly well-mixed but are instead sequestered deep in the interior, in a sort of "ice mantle" just above the core (e.g., Militzer et al., 2008). Indeed, models such as these are being used to advocate that Uranus and Neptune may actually be "ice giants" rather than gas giants (e.g., Lunine, 1993; Boss, 2002). Because of their lower-mass cores and larger heliocentric distances during accretion, the ratio of nebular gas to solid icy 
planetesimal mass accreted during the formation of Uranus and Neptune was likely much lower than that accreted during the formation of Jupiter and Saturn.

While the giant planets are certainly major reservoirs of water in our solar system (as are, likely, giant planets in other solar systems), additional measurements are clearly needed to more fully understand the $\mathrm{O} / \mathrm{H}$ ratio and the absolute and relative abundances and distribution of water in their atmospheres and deep interiors. Significant new insights could come, for example, from new radio frequency observations of the strong $\mathrm{H}_{2} \mathrm{O}$ rotational transition near $22 \mathrm{GHz}$ to be obtained by the NASA Juno mission, set to orbit Jupiter beginning in 2016. In the meantime, researchers will continue to use computational methods to incorporate new and existing telescopic and spacecraft measurements and new laboratory data on the behavior of molecular species and ices at high temperatures and pressures into an improved understanding of water in the giant planets.

\subsubsection{Outer Solar System Satellites and Rings.}

Telescopic and spacecraft spectroscopic observations have shown that all outer solar system satellites except for tidally-heated and devolatilized Io have (or are dominated by) water ice on their surfaces (e.g., Clark et al., 1986). Bulk density information is available for most of these objects from close spacecraft flyby determinations of their masses; the relatively low density values (except for Io) suggest that all of these objects have significantly high water:rock ratios in their interiors. Observations of the ring systems of Saturn, Uranus, and Neptune also reveal the presence of water ice, mixed to varying degrees with silicate dust and low-albedo organic solids (e.g., Sicardy, 2005). Overall, the satellites and rings of the outer solar system represent a significant reservoir of water and is consistent with formation of these bodies beyond the snow line, in a nebular environment dominated by ice-rich planetesimals.

3.2.3. Main Belt and Outer Solar System Asteroids, Comets, and Trans-Neptunian Objects.

\subsubsection{Main Belt Asteroids.}

Early "mapping" of the composition of the main asteroid belt using reflectance spectroscopy and a small number of spacecraft flyby encounters revealed an overall gradient in composition with heliocentric distance that appears consistent with the standard model of solar system formation: rocky and metallic ("igneous") inner solar system bodies transitioning to rocky/icy ("metamorphic"), and then ice-dominated bodies ("primitive") farther out. (e.g., Jones et al., 1990; Gaffey et al., 2002). While the known population of small bodies has grown by orders of magnitude recently (currently $\mathrm{N} \sim 5 \times 10^{5}$ ) because of new surveys and instruments (Minor Planet Center, 2010), and many new dynamical complexities among asteroid populations have been revealed (e.g., Bottke et al., 2002), the overall trend in composition remains an important observational constraint. For example, while the C-type main belt asteroids (which show spectroscopic evidence of hydration as well as similarities to carbonaceous chondrite meteorites) are found throughout the main belt, their distribution peaks in the outer main belt beyond the snow line $(\sim 2.6-3.5 \mathrm{AU}$; e.g., Nelson et al., 1993; Rivkin et al., 2002a). 


\subsubsection{Outer Main Belt and Trojan Asteroids.}

The physical and compositional characteristics of outer main belt asteroids (dominated by D-class objects) and "special" outer solar system asteroid populations like Jupiter's Trojan asteroid clouds ( $\mathrm{N}>4000$; most near 5 AU; Dotto et al., 2008) and the Centaurs (N 250; most with perihelia between Saturn and Neptune; Barucci et al., 2008) are still much less well known compared to inner main belt and near-Earth asteroids. Many members of this population appear to have relatively featureless, red-sloped visible to near-IR spectra consistent with refractory and/or mixed refractory-organic (macromolecular hydrocarbons) surface compositions (e.g., Luu et al., 1994; Dotto et al., 2008; Barucci et al., 2008), in some ways similar to the few available spectra of comet nuclei (e.g., Jewitt and Luu, 1990; see also §3.2.3.3). That is, the lack of spectroscopic evidence for ices, organics, and other volatiles in D-type and Trojan asteroids also occurs for comet nuclei, whose bulk compositions are known to be icy but masked by a thin, dark, refractory mantling layer. A few Centaurs show evidence of water and other ices in their surface spectra (e.g., (5145) Pholus: Cruikshank et al., 1998), and/or intermittent comet-like coma activity and release of water and other volatiles (e.g., (2060) Chiron: Luu and Jewitt, 1990; Luu et al., 1990; Barucci et al., 2008). The relatively small population of known Centaurs is still poorly characterized, however. Whether or not D-type and Trojan asteroids represent a significant reservoir of water is an unresolved question that could require future up-close spacecraft missions to resolve. Given the dynamical importance of the Trojans, in particular, as "trapped" examples of potentially once more distant objects scattered into the inner solar system by the migration of the giant planets (e.g., Tsiganis et al., 2005; Morbidelli et al., 2005), understanding their composition could provide significant insights about the role of external delivery of water to Earth and the other terrestrial planets (see also $\S 4.1$ ).

\subsubsection{Comets.}

Cometary comae reveal prodigious evidence for water vapor and water ice, and modeling of the available data on water and dust abundances suggests water mass fractions around $80 \%$ for typical comet nuclei (e.g., Festou et al., 2004; Encrenaz, 2008). Until very recently, however, water ice had not been directly measured on comet nuclei; as mentioned above, their surfaces were instead observed to be apparently mantled by a dark, reddish layer of refractories and/or macromolecular hydrocarbons. The only direct measurement of comet nucleus water ice comes from the Deep Impact mission's 2005 impact excavation of water ice from the subsurface of comet 9P/Tempel 1 (e.g., Sunshine et al., 2006), which provided support for the hypothesis that the dark, refractory mantles of comet nuclei (and potentially other dark, reddish, primitive objects like D-type and Trojan asteroids) are relatively thin. Four comets (1P/Halley, C/1996 B2 Hyakutake, $\mathrm{C} / 1995$ O1 Hale-Bopp, 8P/Tuttle) have also had their $\mathrm{D} / \mathrm{H}$ ratio in water reported in spectra of their comae (Horner et al., 2007; Villanueva et al., 2009). In all cases D/H was measured to be about 3 times that of Earth's standard mean ocean water D/H value, suggesting that comets and perhaps other external sources may not be the origin of the water on Earth or the other terrestrial planets (see $\S 4.1$ ).

\subsubsection{Trans-Neptunian (Kuiper Belt) Objects.}

The Kuiper Belt extends from $\sim 30$ to $\sim 55$ AU. Water ice has been identified in the spectra of many of the more than 1100 known Trans-Neptunian objects, such as Pluto's 
moon Charon, (50000) Quaoar, (90482) Orcus, 1996 TO66, (136108) Haumea, (10199) Chariklo and others (e.g., see recent review in Barucci et al., 2008). Pluto itself has been found to exhibit a minor water ice component, but its spectrum is dominated by other ices (e.g., $\mathrm{CH}_{4}, \mathrm{~N}_{2}, \mathrm{CO}, \mathrm{CO}_{2}$; e.g., Olkin et al., 2007). Spectroscopic observations of these faint objects are challenging, and so characterization of the surface compositions of these objects is far from complete. Nonetheless, even the very incomplete census of this population so far shows it to be a reservoir rich in volatile ices, including water.

\subsection{Is there water in places where we might not have expected it?}

The common wisdom, according to the standard model of solar system formation, is that only rocky and metallic bodies should condense and accrete into planets inside a solar system's snow line. The expectation, then, is that the terrestrial planets in our solar system should be essentially dry objects. In a bulk sense, the densities of the terrestrial planets confirm this expectation. However, these objects are not-or perhaps were notcompletely water-free. Indeed, even the relatively small fractional abundances of water on these planetary bodies has important implications for planetary evolution and, of course, life.

\subsubsection{Airless Inner Solar System Bodies.}

Mercury and the Moon are rocky/metallic and intrinsically dry planets. However, theoretical modeling and some radar and orbital remote sensing observations have revealed that water ice and other volatiles could potentially exist within permanently-shadowed regions near their poles (e.g., Harmon et al., 1994) because of these bodies' relatively low orbital inclinations. Confirmation that the radar-bright regions near Mercury's poles are water ice deposits is a major measurement objective of the MESSENGER mission, set to enter Mercury orbit in 2011. NASA's recent LCROSS mission impacted an Atlas rocket upper stage into one of these permanently-shadowed regions near the Moon's south pole. The resulting ejecta plume was observed to contain water vapor, $\mathrm{OH}$, and other volatiles, presumably deposited by cometary impacts (e.g., Colaprete et al., 2009). Although the observed abundances were very small, the detection confirms the hypothesis that volatiles like water can exist stably in special places even on the harsh lunar surface, and thus provide samples of (potentially ancient) cometary materials for scientific study, as well as potential resources for future human explorers. Spectroscopic observations of Phobos, Deimos (e.g., Rivkin et al., 2002b), and some of the members of the Aten, Apollo, and Amor near-Earth asteroid populations $(\mathrm{N} \sim 6700$ at present) have not yet revealed any specific evidence of water ice or other volatiles among any of these small inner solar system bodies, although many of these objects are C-type or other spectroscopic classes that could be expected to contain some volatile component (e.g., Binzel et al., 2002).

\subsubsection{Venus.}

Because of its extremely high temperatures, the surface and (likely) interior of Venus are dry, and the planet's atmosphere contains only a minor trace amount of water vapor. However, the measured $\mathrm{D} / \mathrm{H}$ ratio in the atmosphere is some 150 times higher than that of the Earth's oceans, strongly suggesting that there has been a substantial amount of water that has escaped over the planet's history (e.g., Donahue and Russell, 1997). This raises many questions: how did the water escape (slowly? catastrophically?), and what was the origin and abundance of the putative early reservoir of water on Venus? 
Answering these questions and solving the puzzle of water of Venus will likely require future (challenging) orbital, landed, and perhaps even sample return missions.

\subsubsection{Mars.}

Mars is the most Earth-like of the other terrestrial planets, and it is the only other one with significant quantities of water stably existing on its surface-primarily as ice in the polar caps, but also as a small amount of atmospheric water vapor. Recent orbital and surface lander measurements have also revealed significant quantities of ice in the shallow high-latitude subsurface (e.g., Boynton et al., 2008; Feldman et al., 2008; Smith et al., 2009). Given its distance closer to (but still well within) the snow line and lower density, Mars might be expected to contain a higher bulk water component than the other terrestrial planets. However, modeling, geochemical data from Martian meteorites and from surface and orbital measurements suggest that the Martian mantle is likely to be dry (e.g., McSween, 1994; Lunine et al., 2003; McSween et al., 2009). The planet's relatively small core and thus higher ratio of rocky to metallic materials would thus presumably explain the lower density compared to the other terrestrial planets. The Martian surface today is a cold (mean temp $\sim 215 \mathrm{~K}$ ), low pressure $(5-10 \mathrm{mbar}$ ), and hyperarid environment (Baker, 2001). However, there is ample evidence that liquid water once existed stably on the Martian surface (and likely, subsurface), and thus that the temperature and atmospheric pressure were likely higher. The evidence includes geologic landforms like river channels and deltas that are preserved in some of the planet's most ancient (3-4 Ga) terrains; large regions of (ancient) heavily-eroded terrain suggesting the past action of precipitation and surface runoff; and a variety of hydrated minerals, including clays, sulfates, evaporite salts, and certain iron oxides, that can only have formed by the alteration of precursor minerals in an aqueous solution (see detailed reviews in, e.g., Carr, 1996; Bell, 2008). The discovery that Mars once had liquid water raises intriguing possibilities about the potential emergence of life on the Red Planet. However, it also raises perplexing questions that are the focus of much of the ongoing spacecraft exploration of Mars: What was the source of early water on Mars? Where did the water go: subsurface? slow escape to space? catastrophic loss via impacts? And - from the standpoint of habitability - how long was the environment able to support liquid water stably on the surface?

\subsubsection{Earth.}

We take the presence of water on our home planet for granted because more than $70 \%$ of Earth's surface area is covered by water (equal to a global surface layer $\sim 3 \mathrm{~km}$ deep, or about $0.02 \%$ of Earth's mass). However, from a cosmochemical perspective, the bulk composition of the Earth is estimated to be only $<0.1$ wt.\% water (e.g., Drake and Righter, 2002). Earth's thin layer of surface water cloaks what is truly a dry world. Where then did Earth's surface water come from? We return to this question in $\S 4.1$ below. Despite its small relative abundance, water has dramatic effects on our planet's geology, geochemistry, climate, and biology. For example, the oceans sequester enormous quantities (tens of bars or more) of $\mathrm{CO}_{2}$ in solution, removing a potential source of additional greenhouse warming from the atmosphere (e.g., Walker et al., 1981). Water is also thought to play a major role in the lubrication of Earth's several dozen lithospheric plates, enabling the subduction that eventually leads to melting and recycling of crustal rocks and some of the sequestered atmospheric $\mathrm{CO}_{2}$ (e.g., Ragenauer-Lieb et al., 2001). Water is responsible for erosion of surface landforms and alteration of minerals to 
oxidized and/or hydrated phases. And as discussed in $\S 2$ above and $\S 5$ below, liquid water is also a critical component of life on this planet.

\section{The Special Appeal of Liquid Water}

\subsection{Origin of Earth's Water?}

Because of the critical role of water in the past and present geologic and biologic evolution of our planet and probably other Earth-like planets, as well as the expectation from classical models of solar system formation that the Earth should not have formed with a significant inventory of water, there is a special interest in understanding the enigma of our planet's inventory of water. As outlined in many recent studies and reviews (e.g., Morbidelli et al., 2000; Drake and Righter, 2002; Lunine et al., 2003; Drake, 2005; Owen, 2008; Albarede, 2009; Holland et al., 2009), the primary hypotheses being explored for Earth's water are split between those that invoke endogenic sources, with water originating from the population of planetesimals accreting in near-Earth space to form our planet, versus those that invoke exogenic sources, with water having been brought in from bodies farther out in the solar system, likely beyond the snow line. Endogenic hypotheses posit that the early Earth intrinsically formed with a significant internal component of water that, through volcanic and/or impact degassing over time, is the major source of our planet's surface water. Exogenic hypotheses invoke large early impacts of the proto-Earth with volatile-bearing planetesimals or the addition of a "late veneer" (post Moon-forming event) of water from comet and asteroid impacts. Endogenic and exogenic hypotheses have strengths and weaknesses based on available data and current modeling of solar system formation. For example, the concept of Earth accreting from "wet" planetesimals would appear to require that volatile-rich source material should have been part of the early accretion of the planet, even though most of that material may have only been available beyond the solar system's snow line (which may have been out near 4-5 AU early in the history of the solar system; e.g., Stevenson and Lunine, 1988). There are issues with the potential sources of externally-delivered water as well. As mentioned above, the measured D/H ratio in comets is inconsistent with the value in Earth's oceans, arguing against a cometary source. However, there are still important questions about whether the small number of available cometary $\mathrm{D} / \mathrm{H}$ measurements truly sample the population's values; whether the D/H ratio of Earth's oceans could have changed over time due to dilution, weathering, or other processes; and whether the assumed bulk D/H ratio of the Earth overall-and indeed the bulk abundance of water in the Earth-is truly known (e.g., Abe et al., 2000; Drake and Righter, 2002; Smyth et al., 2006). There are also unresolved questions about the ability of near-Earth and main belt planetesimals and asteroids-primitive materials in the source region of the accreting Earth-to explain Earth's water inventory. The parent bodies of ordinary chondrite meteorites could contain from 0.1 to as much as 9 wt. $\% \mathrm{H}_{2} \mathrm{O}$ by some estimates (e.g., Zinner, 1988). The higher ranges of these estimated abundances could be adequate to justify Earth's water inventory as intrinsic rather than extrinsic, and the ordinary chondrites also have $\mathrm{D} / \mathrm{H}$ ratios comparable to that of Earth's oceans (e.g., Drake and Righter, 2002). However, the issue of whether the ordinary chondrites as opposed to other primitive source materials such as the carbonaceous chondrites represent the building block materials for terrestrial planet accretion remains controversial (e.g., Morbidelli et al., 2000). Recently it has been proposed that outer main belt and outer solar system asteroids (large compared to comet nuclei, and hydrated because of their formation beyond the snow line) could have been 
significant sources of Earth's water during and shortly after accretion. More specifically, the hypothesized migration of the giant planets during the early history of the solar system has been invoked as a source of substantial dynamical mixing/scattering of small bodies from the outer solar system to the inner solar system, and as a potential explanation for the late heavy bombardment of the Earth-Moon system that is preserved in the lunar impact record (e.g., Gomes et al., 2005; Tsiganis et al., 2005; Morbidelli et al., 2005). In this so-called "Nice model" of solar system evolution, the orbits of many small outer solar system objects could have been dramatically altered when Jupiter and Saturn passed through a 2:1 mean-motion resonance around $\sim 4 \mathrm{Ga}$, scattering relatively large, volatile-rich bodies into the inner solar system (as well as the Kuiper Belt) and providing potential large-impact sources of water for the terrestrial planets. Interestingly, it may be possible to test the Nice model by confirming the volatile-rich compositions of Jupiter's Trojan asteroids-a large population of small bodies that has been trapped in Jupiter's Lagrange points since the end of the proposed period of giant planet migration (e.g., Dotto et al., 2008). It could be argued that invoking cometary and/or asteroidal impacts to deliver Earth's water relies on ad hoc or stochastic events or assumptions. However, the apparent importance of stochastic impact events in the evolution of the solar system is now more appreciated than ever, with serious impact-related hypotheses being explored to explain, for example, the origin of the Moon, the late heavy bombardment, and the unusual spin properties of Uranus and Venus (e.g., Korycansky et al., 1990; Canup and Asphaug, 2001; Gomes et al., 2005; Davies, 2008). While the origin of Earth's water is still a highly debated and exciting topic of study, it is also of course entirely possible that a combination of both endogenic and exogenic sources could explain our planet's current surface water inventory.

\subsection{Habitable Zones and Water on Other Planets}

The habitable zone is a key concept or metric that enables astronomers to think more broadly about the concept of water on any planets-whether in our solar system or others. Specifically, the habitable zone is defined as the heliocentric distance from a star within which conditions would be favorable for life as we know it to exist (e.g., Hart, 1979). Even more specifically, the definition provides a guide to assess habitability (the potential to develop and sustain life) by providing an estimate of the planetary region where liquid water could be stable on a planet with Earth-like surface conditions. The formal definition of the habitable zone defines the mid-line of the zone at a heliocentric distance in $\mathrm{AU}$ of $\left(\mathrm{L}_{\text {star }} / \mathrm{L}_{\text {sun }}\right)^{0.5}$, where $\mathrm{L}$ is the stellar or solar luminosity. The inner and outer boundaries of the habitable zone are typically assumed to range from about $95 \%$ to $135 \%$ of the midline, based mostly on analogy with our own solar system. However, the boundaries are widely recognized to be fuzzy, and the entire definition itself is understood to be significantly biased by the particular characteristics of our own solar system-as yet the only place where a habitable environment is known to exist. While the concept of the habitable zone is a useful guide and starting point for understanding the role of water in influencing a planet's potential biologic and even geologic evolution, even in our own solar system there are clearly exceptions to this "rule." Prominent among these exceptions are Jupiter's moon Europa and Saturn's moon Enceladus, both of which are well outside the classical habitable zone but both of which exhibit compelling evidence for liquid water just below their icy crusts. Evidence for a deep ocean on Europa comes from Voyager and Galileo mission geologic evidence of thin icy crustal plates floating and moving over a liquid layer, salty mineral deposits extruded onto the surface, and gravity and magnetic field measurements that suggest a salty, conducting liquid subsurface layer (e.g., Carr 
et al., 1998; McCord et al., 1999; Kivelson et al., 2000). The surprising evidence for liquid water under the icy crust of tiny Enceladus $(\mathrm{R} \sim 250 \mathrm{~km})$ comes from Cassini mission observations of plumes of water ice emanating from warmer fractures near the satellite's south pole (e.g., Porco et al., 2006). In the case of Europa, tidal heating from Jupiter provides the energy source that would prevent a subsurface ocean from freezing solid; the energy source powering Enceladus's geysers is presently unknown, but possibilities include tidal heating from Saturn and other satellites, and/or radioactive heating from rocky materials deep within the satellite. Beyond our solar system, the study of extrasolar planets has expanded rapidly over the past decade, with more than 400 extrasolar planets currently known, and more being announced frequently (see, e.g., http://exoplanet.eu/). Even more exciting is that the first planets are now being discovered within the habitable zones around other nearby, Sun-like stars (e.g., Selsis et al., 2007; von Bloh et al., 2007). Water vapor and water ice are now being detected in circumstellar disks and even the atmospheres of some exoplanets (e.g., Watson et al., 2007; Tinetti et al., 2007). The study of water in exoplanets and of the nature of extrasolar habitable zones is just beginning. It's exciting to realize that astronomers and planetary scientists are on the cusp of some significant new discoveries including, soon, the expected discovery of the first truly Earth-like planets within the habitable zones of other stars.

\section{Implications: Why care about Water on Planets?}

Our own solar system provides a warning that we must interpret concepts like the habitable zone with care: special environments outside the classically-defined habitable zone could still satisfy the constraints of habitability for life as we know it (and, of course, who knows about life as we don't know it?). An additional caveat is that a star's habitable zone should be expected to evolve with time as the star evolves. For example, the Sun's habitable zone has moved outwards as the solar luminosity has increased over time (e.g., Kasting and Catling, 2003). This implies that Venus was likely to have been solidly within the habitable zone early in the history of the solar system when the Sun was fainter, and that Mars will move solidly into the habitable zone in the far future as solar luminosity increases. If the Sun continues to evolve as expected, the inner edge of the solar system's habitable zone will eventually sweep past the Earth (in perhaps $\sim 1 \mathrm{~Gy}$ ), vaporizing the oceans, releasing trapped $\mathrm{CO}_{2}$ into the atmosphere, and potentially rendering our planet as inhospitable as Venus is today. While prognosticating about a planet's distant future is fraught with assumptions and uncertainties, the implications of this particular prediction are so drastic that it can't help but to compel us to learn more about stellar and planetary evolution, and about the role that water and other volatiles play in the evolution of a planet's climate.

Studying the triad of Venus, Earth, and Mars has yielded particularly important and illuminating insights about the critical role of the physical state of water in influencing the evolution of a terrestrial planet. In particular, water appears to have played a major role in the divergent evolutions of these three planets. In the standard model of solar system formation, all three planets would have formed with approximately the same starting (primarily rocky/metallic) bulk composition, and with generally thin and reducing $\left(\mathrm{H}_{2}, \mathrm{CH}_{4}\right.$, etc.) atmospheres because of their relatively poor ability to retain nebular gas and their location in the relatively volatile-poor inner solar system. Differentiation, outgassing, and additional late heavy bombardment and subsequent accretion of volatiles would have resulted in the buildup of $\mathrm{CO}_{2}, \mathrm{H}_{2} \mathrm{O}, \mathrm{SO}_{2}, \mathrm{~N}_{2}$, etc. in their atmospheres over time, while $\mathrm{H}_{2}$ would have slowly been lost due to escape and solar wind breakdown. At the heliocentric distance of Venus, high temperatures kept the water in the vapor 
phase, while at Mars's distance the water would primarily have existed as ice (even if the past atmosphere had been thick enough to allow liquid water to occasionally be stable on the surface). Earth's surface conditions were, of course, just right for liquid water to be stable, leading to the (early) formation of deep oceans. Earth's oceans provide a sink for atmospheric greenhouse gases like $\mathrm{CO}_{2}$ and $\mathrm{SO}_{2}$ (and of course, water vapor), either directly dissolved in water or precipitated as carbonate and sulfate rocks and stored in crustal reservoirs. The lack of oceans on Venus (currently, at least) means that the $\mathrm{CO}_{2}$ was not sequestered and thus became the source of a massive amount of runaway greenhouse warming. And while there is good evidence for the past presence of liquid water on Mars, the lack of evidence for extensive, global-scale carbonate deposits (despite detailed searches from orbital and landed missions) argues against the planet ever having had long-lived, large-scale oceans. While $\mathrm{CO}_{2}$ still dominates the Martian atmosphere, it is currently in too small an abundance to provide any significant greenhouse warming. If Mars still harbors significant amounts of water, it is likely locked up as polar and subsurface ice.

The ongoing discovery of the role of water $\left(\right.$ and $\left.\mathrm{CO}_{2}\right)$ in the surfaces, atmospheres, and climates of Venus and Mars provides global-scale context and insight about the short- and long-term influences of these volatiles on our home planet. Indeed, NASA and other space agencies have recently adopted a "follow the water" theme for the exploration of Mars and other destinations in our solar system as well as the study of extrasolar planets. As we have seen from previous telescopic and spacecraft observations as well as laboratory and modeling studies, to follow the water focuses us on understanding key aspects of solar system formation, planetary evolution, and planetary atmospheric, surface, and interior processes all under a single theme that is both scientifically rewarding as well as interesting and exciting to the general public. Ultimately, to search for water is to search for ourselves.

\section{Acknowledgements}

I am grateful to Martha Haynes and other colleagues on the IAU Executive Committee for inviting me to present this review as an Invited Discourse at the IAU's General Assembly XXVII in Rio de Janeiro in August, 2009. I am also extremely grateful to Jonathan Lunine, Amara Graps, Dale Cruikshank, and Jamie Lloyd for providing background information, preprints, and general collegial support for my research into this exciting and timely topic. My research on the past history and present inventory of water on specific planets and other planetary bodies has been supported over the years by grants and contracts from the NASA Planetary Geology, Planetary Astronomy, and Mars Data Analysis Programs, from the Jet Propulsion Laboratory/Caltech, and from the Space Telescope Science Institute.

\section{References}

Abe, Y., E. Ohtani, T. Okuchi, K. Righter, \& M. J. Drake "Water in the early Earth", in "Origin of the Earth and Moon", edited by R. M. Canup and K. Righter, Univ. of Ariz. Press, Tucson, 2000 pp. 413-433

Albarède, F. Volatile accretion history of the terrestrial planets and dynamic implications, Nature, 461, 1227-1233, 2009.

Atreya, S. K., Atmospheres and Ionospheres of the Outer Planets and Their Satellites, 234 pp., Springer-Verlag, Berlin, 1986.

Baker, V. R., Water and the martian landscape, Nature, 412, 228-236, 2001. 
Barucci, M. A., "Composition and Surface Properties of Transneptunian Objects and Centaurs" in The Solar System Beyond Neptune, M. A. Barucci, H. Boehnhardt, D. P. Cruikshank, and A. Morbidelli (eds.), University of Arizona Press, Tucson, pp. 143-160, 2008.

Bell III, J. F. (Editor) The Martian Surface: Composition, Mineralogy, and Physical Properties, Cambridge University Press (ISBN-13: 9780521866989), Cambridge, 688 pp., 2008.

Binzel, R. P., D. F. Lupishko, M. DiMartino, R. J. Whiteley, \& G. J. Hahn, "Physical Properties of Near-Earth Asteroids", in Asteroids III (W.F. Bottke Jr. et al., eds.), pp. 255-271, University of Arizona Press., 2002.

Boss, A. P., Formation of gas and ice giant planets, Earth Planet. Sci. Lett., 202, 513-523, 2002.

Bottke, W. F., A. Cellino, P. Paolicchi, \& R. P. Binzel (editors), Asteroids III, Univ. Arizona Press, $785 \mathrm{pp}$.

Boynton, W. V., G. J. Taylor, S. Karunatillake, R. C. Reedy, \& J. M. Keller, "Elemental abundances determined via the Mars Odyssey GRS", Chapter 5 in "The Martian Surface: Composition, Mineralogy, and Physical Properties" (J.F. Bell III, editor), Cambridge Univ. Press, 105-124, 2008.

Canup, R. M. \& E. Asphaug, Origin of the Moon in a giant impact near the end of the Earth's formation, Nature, 412, 708-712, 2001.

Carr, M. H., Water on Mars, Oxford Univ. Press, 248 pp., 1996.

Carr, M. H., Belton, M. J. S., Chapman, C. R., Davies, M. E., Geissler, P., Greenberg, R., McEwen, A. S., Tufts, B. R., Greeley, R., Sullivan, R., Head, J. W., Pappalardo, R. T., Klaasen, K. P., Johnson, T. V., Kaufman, J., Senske, D., Moore, J., Neukum, G., Schubert, G., Burns, J. A., Thomas, P., \& Veverka, J., Evidence for a subsurface ocean on Europa, Nature, 391, 363-365, 1998.

Cassen, P., Utilitarian models of the solar nebula, Icarus 112, 405-429, 1994.

Chambers, J. E., Planetary accretion in the inner Solar System, Earth Planet. Sci. Lett., 223, 241-252, 2004.

Clark, R. N., Water frost and ice: The near-infrared spectral reflectance 0.65-2.5 m, J Geophys. Res., 86, 3087-3096, 1981.

Clark R. N., Fanale F. P., \& Gaffey M. J., "Surface composition of natural satellites", in "Satellites", ed. J.A. Burns and M.S. Matthews, pp. 437-491. Tucson: Univ. Arizona Press, 1986.

Colaprete, A., Briggs, G., Ennico, K., Wooden, D., Heldmann, J., Sollitt, L., Asphaug, E., Korycansky, D., Schultz, P., Christensen, A., Galal, K., Bart, G. D., \& the LCROSS Team, An Overview of the Lunar Crater Observation and Sensing Satellite (LCROSS) Mission Results from Swing-by and Impact, Lunar Exploration Analysis Group, November 16-19, Houston, Texas. LPI Contribution No. 1515, p. 11, 2009.

Cruikshank, D. P., T. L. Roush, M. J. Bartholomew, T. R. Geballe, Y. J. Pendleton, S. White, J. F. Bell III, J. K. Davies, T. C. Owen, C. deBergh, D. Tholen, M. P. Bernstein, R. H. Brown, K. A. Tryka, \& C. M. Dalle Ore, The composition of Centaur 5145 Pholus, Icarus, 135, 389-407, 1998.

Davies, J. H., Did a mega-collision dry Venus' interior? Earth Planet. Sci. Lett., 268, 376-383, 2008.

de Bergh, C., B. Schmitt, L. V. Moroz, E. Quirico, \& D. P. Cruikshank, "Laboratory Data on Ices, Refractory Carbonaceous Materials, and Minerals Relevant to Transneptunian Objects and Centaurs", in "The Solar System Beyond Neptune", M. A. Barucci, H. Boehnhardt, D. P. Cruikshank, and A. Morbidelli (eds.), University of Arizona Press, Tucson, pp. 483-506, 2008.

Donahue, T. M. \& C. T. Russell, "The Venus Atmosphere and Ionosphere and their Interactions with the Solar Wind: An Overview," in "Venus II", Univ. Arizona Press, pp. 3-31, 1997.

Dotto, E., J. P. Emery, M. A. Barucci, A. Morbidelli, \& D. P. Cruikshank, "De Troianis: The Trojans in the planetary system," in "The Solar System Beyond Neptune" (Barucci, Boehnhardt, Cruikshank, Morbidelli, Eds.), pp. 383-396, University of Arizona Press, Tucson, 2008.

Drake, M. J., Origin of water in the terrestrial planets, Met. \& Plan. Sci., 40, 519-527, 2005.

Drake, M. J. \& Righter K., Determining the composition of the Earth, Nature, 416, 39-46, 2002.

Encrenaz, T., Water in the solar system, Ann. Rev. Astron. Astrophys., 46, 57-87, 2008.

Feldman, W. C., M. C. Mellon, O. Gasnault, S. Maurice, \& T. H. Prettyman, "Volatiles on Mars: Scientific results from the Mars Odyssey Neutron Spectrometer", Chapter 6 in "The 
Martian Surface: Composition, Mineralogy, and Physical Properties (J.F. Bell III, editor)", Cambridge Univ. Press, 125-152, 2008.

Festou, M. C., H. U. Keller, \& H. A. Weaver (editors), Comets II, Univ. Arizona Press, 745 pp., 2004.

Feuchtgruber, H., E. Lellouch, T. de Graauw, B. Bézard, T. Encrenaz, \& M. Griffin, External supply of oxygen to the atmospheres of the giant planets, Nature, 389, 159-162, 1997.

Gaffey, M. J., E. A. Cloutis, M. S. Kelley, \& K. L. Reed, "Mineralogy of Asteroids", in Asteroids III (W.F. Bottke Jr. et al., eds.), pp. 653-667. University of Arizona Press., 2002.

Gomes, R., H. F. Levison, K. Tsiganis, \& A. Morbidelli, Origin of the cataclysmic late heavy bombardment period of the terrestrial planets, Nature 435, 466-469, 2005.

Grundy, W. M., M. W. Buie, J. A. Stansberry, J. R. Spencer, \& B. Schmitt, Near-Infrared Spectra of Icy Outer Solar System Surfaces: Remote Determination of H2O Ice Temperatures, Icarus, 142, 536-549, 1999.

Harmon, J. K., Slade, M. A., Vélez, R. A., Crespo, A., Dryer, M. J., \& Johnson, J. M., Radar mapping of Mercury's polar anomalies, Nature, 369, 213-215, 1994.

Hart, M. H., Habitable zones about main sequence stars, Icarus, 37, 351-357, 1979.

Holland, G., M. Cassidy, \& C. J. Ballantine, Meteorite Kr in Earth's mantle suggests a late accretionary source for the atmosphere, Science, 326, 1522-1525, 2009.

Horner, J., O. Mousis, \& F. Hersant, Constraints on the Formation Regions of Comets from their D:H Ratios, in Earth, Moon, Planets, 100, 43-56, 2007.

International Astronomical Union, "IAU 2006 General Assembly: Resolutions 5 and 6", http://www.iau.org/static/resolutions/Resolution_GA26-5-6.pdf, Aug. 24, 2006.

Jewitt, D. C. \& J. X. Luu, CCD spectra of asteroids II: The Trojans as spectral analogs of cometary nuclei, Astron. J., 100, 933-944, 1990.

Jones, T. D., L. A. Lebofsky, J. S. Lewis, \& M. S. Marley, The composition and origin of the $C$, $P$, and $D$ asteroids - Water as a tracer of thermal evolution in the outer belt, Icarus, 88, $172-192,1990$.

Kasting, J. \& D. Catling, Evolution of a Habitable Planet, Ann. Rev. Astron. Astrophys., 41, 429-463, 2003.

Kivelson, M. G., K. K. Khurana, C. T. Russell, M. Volwerk, R. J. Walker, \& C. Zimmer, Galileo Magnetometer measurements: A stronger case for a subsurface ocean at Europa, Science, 289, 1340-1343, 2000.

Korycansky, D. G., P. Bodenheimer, P. Cassen, \& J. B. Pollack, One-dimensional calculations of a large impact on Uranus, Icarus 84, 528-541, 1990.

Lecar, M., M. Podolak, D. Sasselov, E. Chiang, On the location of the snow line in a protoplanetary disk, Ap. J., 640, 1115-1118, 2006.

Lellouch, E., B. Bézard, J. I. Moses, G. R. Davis, P. Drossart, H. Feuchtgruber, E. A. Bergin, R. Moreno, \& T. Encrenaz, The Origin of Water Vapor and Carbon Dioxide in Jupiter's Stratosphere, Icarus, 159, 112-131, 2002.

Lunine, J. I., The atmospheres of Uranus and Neptune, Ann. Rev. Astron. Astrophys., 31, 217263, 1993.

Lunine, J. I., J. Chambers, A. Morbidelli, \& L. A. Leshin, The origin of water on Mars, Icarus, $165,1-8,2003$.

Luu, J. X. \& D. C. Jewitt, Cometary activity in 2060 Chiron, Astron. J., 100, 913-932, 1990.

Luu, J. X., D. C. Jewitt, \& E. Cloutis, Near-infrared spectroscopy of primitive Solar System objects. Icarus 109, 133-144, 1994.

Luu, J. X., D. C. Jewitt, \& C. Trujillo, Water Ice in 2060 Chiron and Its Implications for Centaurs and Kuiper Belt Objects, Ap. J., 531, L151-L154, 1990.

Matson, D. L. \& R. H. Brown, Solid-state greenhouses and their implications for icy satellites, Icarus, 77, 67-81, 1989.

McCord, T. B., G. B. Hansen, D. L. Matson, T. V. Johnson, J. K. Crowley, F. P. Fanale, R. W. Carlson, W. D. Smythe, P. D. Martin, C. A. Hibbitts, J. C. Granahan, \& A. Ocampo, Hydrated salt minerals on Europa's surface from the Galileo near-infrared mapping spectrometer (NIMS) investigation, J. Geophys. Res., 104, 11827-11852, 1999.

McSween H. Y., What we have learned about Mars from SNC meteorites, Meteoritics, 29, 757$779,1994$. 
McSween, H. Y., G. J. Taylor, \& M. B. Wyatt, Elemental composition of the Martian crust, Science, 324, 736-739, 2009.

Militzer, B., W. B. Hubbard, J. Vorberger, I Tamblyn, \& S. A. Bonev, A Massive Core in Jupiter Predicted from First-Principles Simulations, Ap. J., 688, L45-L48, 2008.

Minor Planet Center, IAU/Smithsonian Astrophysical Observatory, lists of minor planets: http://www.cfa.harvard.edu/iau/lists/Lists.html, 2010.

Morbidelli, A., Chambers, J., Lunine, J. I., Petit, J. M., Robert, F., Valsecchi, G. B., \& Cyr, K. E., Source regions and timescales for the delivery of water on Earth, Meteor. Planet. Sci., 35, 1309-1320, 2000.

Morbidelli, A., H. F. Levison, K. Tsiganis, \& R. Gomes, Chaotic capture of Jupiter's Trojan asteroids in the early Solar System, Nature, 435, 462-465, 2005.

Nelson, M. L., D. T. Britt, \& L. A. Lebofsky, Review of asteroid compositions, in Resources of Near-Earth Space, Univ. Arizona Press, pp. 493-522, 1993.

Olkin, C. B., Young, E. F., Young, L. A., Grundy, W., Schmitt, B., Tokunaga, A., Owen, T., Roush, T., \& Terada, H., Pluto's Spectrum from 1.0 to 4.2 ? $m$ : Implications for Surface Properties, Astron. J., 133, 420-431, 2007.

Owen, T., The contributions of comets to planets, atmospheres, and life: Insights from CassiniHuygens, Galileo, Giotto, and inner planet missions, Space Sci. Rev., 138, 301-316, 2008.

Planetary Science Institute, "Petition Protesting the IAU Planet Definition," http://www.ipetitions.com/petition/planetprotest/, 2006.

Podolak, M., The location of the snow line in protostellar disks, Invited talk at IAU Symposium 263: Icy Bodies in the Solar System, Rio de Janeiro, Aug. 2009. arXiv preprint http://arxiv.org/abs/0911.4803v1.

Pollack, J. B., O. Hubickjy, P. Bodenheimer, J. J. Lissauer, M. Podolak, \& Y. Greenzweig, Formation of the Giant Planets by Concurrent Accretion of Solids and Gas, Icarus, 124, 62-85.

Porco, C. C., P. Helfenstein, P. C. Thomas, A. P. Ingersoll, J. Wisdom, R. West, G. Neukum, T. Denk, R. Wagner, T. Roatsch, S. Kieffer, E. Turtle, A. McEwen, T. V. Johnson, J. Rathbun, J. Veverka, D. Wilson, J. Perry, J. Spitale, A. Brahic, J. A. Burns, A. D. Del Genio, L. Dones, C. D. Murray, \& S. Squyres, Cassini Observes the Active South Pole of Enceladus, Science, 311, 1393-1401, 2006.

Ragenauer-Lieb, K., Yuen, D., \& Branlund, J., The initiation of subduction: criticalilty by addition of water? Science, 294, p. 578-580, 2001.

Rivkin, A. S., E. S. Howell, F. Vilas, \& L. A. Lebofsky, "Hydrated Minerals on Asteroids: The Astronomical Perspective," in Asteroids III (W.F. Bottke Jr. et al., eds.), pp. 235-253, University of Arizona Press., 2002.

Rivkin, A. S., R. H. Brown, D. E. Trilling, J. F. Bell III, \& J. H. Plassmann, Near-infrared spectrophotometry of Phobos and Deimos, Icarus, 156, 64-75, 2002.

Selsis, F., J. F. Kasting, B. Levrard, J. Paillet, I. Ribas, \& X. Delfosse, Habitable planets around the star Gliese 581 Astron. Astrophys., 476, 1373-1387, 2007.

Sicardy, B., Dynamics and Composition of Rings, Space Sci. Rev., 116, 457-470, 2005.

Smith, P. H. and 35 others, H2O at the Phoenix landing site, Science, 325, 58-61, 2009.

Smyth, J. R., D. J. Frost, F. Nestola, C. M. Holl, \& G. Bromiley, Olivine hydration in the deep upper mantle: Effects of temperature and silica activity, Geophys. Res. Lett., 33, L15301, 2006.

Stevenson, D. J. \& J. I. Lunine, Rapid formation of Jupiter by diffuse redistribution of water vapor in the solar nebula, Icarus, 75, 146-155, 1988.

Sunshine, J., A’Hearn, M. F.; Groussin, O.; Li, J.-Y.; Belton, M. J. S.; Delamere, W. A.; Kissel, J.; Klaasen, K. P.; McFadden, L. A.; Meech, K. J.; Melosh, H. J.; Schultz, P. H.; Thomas, P. C.; Veverka, J.; Yeomans, D. K.; Busko, I. C.; Desnoyer, M.; Farnham, T. L.; Feaga, L. M.; Hampton, D. L.; Lindler, D. J.; Lisse, C. M.; \& Wellnitz, D. D., Exposed Water Ice Deposits on the Surface of Comet 9P/Tempel 1, Science, 311, 1453-1455, 2006.

Sykes, M., Classifying Planets from a Geophysical Perspective,American Astronomical Society, AAS Meeting \#214, \#237.06; Bull. Amer. Astron. Soc., Vol. 41, p. 740, 2009.

Tinetti, G., A. Vidal-Madjar, M. Liang, J. P. Beaulieu, Y. Yung, S. Carey, R. J. Barber, J. Tennyson, I. Ribas, N. Allard, G. E. Ballester, D. K. Sing, \& F. Selsis, Water vapour in the atmosphere of a transiting extrasolar planet, Nature, 448, 169-171, 2007. 
Tsiganis, K., R. Gomes, A. Morbidelli, \& H. F. Levison, Origin of the orbital architecture of the giant planets of the Solar System, Nature, 435, 459-461, 2005.

Villanueva, G. L., M. J. Mumma, B. P. Bonev, M. A. DiSanti, E. L. Gibb, H. Bhnhardt, \& M. Lippi, A Sensitive Search for Deuterated Water in Comet 8P/Tuttle, Ap. J. Lett., 690, L5-L9, 2009.

von Bloh, W., C. Bounama, M. Cuntz, \& S. Franck, The habitability of super-Earths in Gliese 581, Astron. Astrophys., 476, 1365-1371, 2007.

Walker, J. C. B., Hays, P. B., \& Kasting, J. F., A negative feedback mechanism for the long term stabilization of the Earth's surface temperature, J. Geophys. Res., 86, 9776-9782, 1981.

Warren S. G., Optical properties of snow. Rev. Geophys. 20: 67-89, 1982.

Watson, D. M., C. J. Bohac, C. Hull, W. J. Forrest, E. Furlan, J. Najita, N. Calvet, P. d'Alessio, L. Hartmann, B. Sargent, J. D. Green, K.H. Kim \& J. R. Houck, The development of a protoplanetary disk from its natal envelope, Nature, 448, 1026-1028, 2007.

Wong, M. H., P. R. Mahaffy, S. K. Atreya, H. B. Niemann, \& T. C. Owen, Updated Galileo probe mass spectrometer measurements of carbon, oxygen, nitrogen, and sulfur on Jupiter, Icarus, 171, 153-170, 2004.

Zinner, E., "Interstellar cloud material in meteorites", in "Meteorites and the Early Solar System" (ed. Kerridge, J.) Univ. Arizona Press, Tucson, pp. 956-983, 1988. 